\title{
Interaction of Heart Rate and Respiration in Newborn Babies
}

\author{
T. Ä̈rimaa, R. Oja, K. Antila, and I. Välimäki \\ Department of Pediatrics and Cardiorespiratory Research Unit, University of Turku, Turku, Finland
}

\begin{abstract}
Variability of heart rate (HRV) and transthoracic electric impedance respirogram (TEZ) were examined by spectral analysis in three groups of neonates: healthy term babies (22), healthy preterm babies (21), and preterm babies with respiratory distress syndrome (RDS) (11). Heart rate, TEZ, $\mathrm{P}_{\mathrm{tc}} \mathrm{O}_{2}$, and $\mathrm{P}_{\mathrm{tc}} \mathrm{CO}_{2}$ were monitored during quiet sleep on the 1st, 3rd, and 5th day of postnatal life. Autospectra for trend-corrected segments of heart rate and TEZ as well as their cross-spectra and coherence spectra were computed. The peak of HRV spectral density was in $<0.2 \mathrm{~Hz}$ [low frequency (LF)] area ( $<12$ cycles/ $\mathrm{min}$ ) in all the neonates. Intergroup comparisons of average band-integrated spectra revealed that the LF spectral density of HRV was greater in the term babies than in the preterm babies on day 3 . In the babies with RDS, both LF and high-frequency $(\mathrm{HF},>0.2 \mathrm{~Hz}$ ) were abnormally low throughout the study. In the term infants, the TEZ amplitude spectrum was flat on day 1 . On later days, a peak corresponding to the average respiratory rate emerged. In the healthy preterm babies, there was a LF peak in TEZ autospectrum on all days. In the babies with RDS, the peak of ventilator frequency was initially present; finally, the respiratory activity accumulated in the $\mathrm{LF}$ area. In the cross-spectra of term babies, there was a LF peak on all days. On day 5, an additional HF peak appeared, representing respiratory sinus arrhythmia. In the healthy preterm babies, only a LF peak was present. In babies with RDS, there were three peaks in the cross-spectra: one in the LF area, another corresponding to the ventilator frequency, and a third one representing the first harmonic of the ventilator frequency. With increasing age, the ventilator-induced peaks disappeared and only LF activity remained. No correlation was found between the spectral patterns of $\mathrm{HRV}$ and cross-spectra and the values of $\mathrm{P}_{\mathrm{tc}} \mathrm{O}_{2}$ and $\mathrm{P}_{\mathrm{tc}} \mathrm{CO}_{2}$. The spectral patterns of $\mathrm{HRV}$ and TEZ indicate that in newborn infants most of the activity is generated by LF mechanisms. Respiratory sinus arrhythmia is detected in term infants when regular breathing becomes organized. In RDS, respiratory sinus arrhythmia HRV is induced by ventilator. In both groups of preterm babies, the $L F$ regulation of $H R V$ seems to be more firmly connected with regulation and mechanics of respiration. (Pediatr Res 24:745-750, 1988)
\end{abstract}

\section{Abbreviations}

HR, heart rate

HRV, heart rate variability

TEZ, transthoracic electric impedance

Received December 28, 1987; accepted August 16, 1988

Correspondence T. Äärimaa, M.D., Cardiorespiratory Research Unit, University of Turku, Turku, Finland.

${ }^{1}$ Supported by grants from The Academy of Finland and Turku University
RDS, respiratory distress syndrome

$P_{t c}$, transcutaneous pressure

$\mathrm{LF}$, low frequency

$\mathrm{HF}$, high frequency

RSA, respiratory sinus arrhythmia

FFT, fast Fourier transform

$\mathrm{Fc}$, frequency cut-off

IHR, instantaneous heart rate

$R R$, respiratory rate

Recently spectral analysis has been applied to the study of HRV (1-3). The principle of Fourier analysis states that any continuous and periodic signal can be described as a sum of sine (and cosine) waves with different frequency and amplitude composition. After subtraction of unnecessary trends from the digitized source signal, a FFT algorithm may be used to compute efficiently the periodic components of HRV. The interpretation of the spectrum is straightforward; it shows the approximate amount of variation in the data for each frequency (4). The spectrum of one signal can also be examined against the power spectrum of another signal. In addition to visual comparison of the two spectra, these signals, e.g. HR and respirogram may be examined by computing cross-covariance or cross-spectrum between the signals. Thus, the magnitude of similar frequencyspecific variability in the signals is exposed. The time specific linearity between the signals can be presented as a coherencespectrum which is a measure of correlation between the variation of two signals at a given frequency.

Since the early 1960's, it has been known that continuous fluctuations in HR are temporarily attenuated in RDS (5). Quantitative featuring of reduced HRV became practical when stochastic point process analysis techniques were applied using digital computers (6). It was shown that the HRV was reduced to one-third or one-fourth of normal values in this condition (7, 8). HRV has been found to contain several major periodic components or oscillations in the range of $0.02-0.2 \mathrm{~Hz}$ in healthy neonates (9). Very little is known about spectral patterns of HRV and cardiorespiratory interactions (10).

As respiration is the best known physiologic interference factor modulating $\mathrm{HR}$, we wanted to evaluate the interrelation of heart rate and respiratory activity using autospectral and cross-spectral analysis techniques to elucidate how much and at which frequency respiration explains HRV in healthy neonates and in infants with RDS.

\section{MATERIALS AND METHODS}

The group of subjects consisted of 22 healthy term infants, 21 healthy preterm infants, and 11 preterm infants with RDS (Table 1). Four of the term infants, 14 of the healthy premature infants, and 6 of the infants with RDS were born by cesarean section. The main reasons for cesarean section were toxemia, asphyxia 
and the policy in our hospital to deliver premature babies under 32 wk of gestational age by cesarean section. The term infants were normally cared for by their mothers on the obstetrical ward. The preterm infants were admitted to the neonatal ward. The healthy preterm infants were initially nursed in incubators.

All the babies with RDS were treated at the newborn intensive care unit. At least for some of their first five days they were treated with intermittent mandatory ventilation positive pressure ventilatory assistance (Baby Bird, Bird Corporation, Palm Springs, CA), but there was also spontaneous respiration. The babies received antibiotics (penicillin-gentamycin) as a routine safety treatment. None had proven sepsis. The preterm infants received 5 to $10 \%$ glucose supplemented with $\mathrm{Na}$ and $\mathrm{K}$ in umbilical arterial or peripheral venous line starting from the volume of $60 \mathrm{ml} / \mathrm{kg} /$ day. They were fed with breast milk in

Table 1. Clinical data of neonates*

\begin{tabular}{|c|c|c|c|}
\hline & Term & Preterm & RDS \\
\hline Girls & 8 & 10 & 3 \\
\hline Boys & 14 & 11 & 8 \\
\hline $\mathrm{GA}<33 \mathrm{wk}$ & & 4 & 6 \\
\hline GA & $38.9(\mathrm{SD} 1.1)$ & $33.8(\mathrm{SD} 2.7)$ & $30.7(\mathrm{SD} 2.6)$ \\
\hline (wk) & $(37-41)$ & $(27-36)$ & $(27-34)$ \\
\hline BW & $3410(\mathrm{SD} 632)$ & $2180(\mathrm{SD} 636)$ & 1664 (SD 779) \\
\hline (g) & $(2210 \dagger-5600)$ & $(1100-3630)$ & $(800-2420)$ \\
\hline Apgar & $\begin{array}{l}8.2(\mathrm{SD} 1.5) \\
(8-10)\end{array}$ & $\begin{array}{l}7.1(\text { SD 2.0) } \\
(2-9)\end{array}$ & $\begin{array}{l}6.3(\mathrm{SD} 1.8) \\
(2-8)\end{array}$ \\
\hline $\begin{array}{l}\mathrm{P}_{\mathrm{tcCO}_{2}} \\
\text { (torr) }\end{array}$ & $\begin{array}{l}48.3(\text { SD } 7.5) \\
(34-60)\end{array}$ & $\begin{array}{l}54.2(\mathrm{SD} 8.6) \\
(39-87)\end{array}$ & $\begin{array}{l}\text { 61.4 (SD 17.4) } \\
(27-101)\end{array}$ \\
\hline $\begin{array}{l}\mathrm{P}_{\mathrm{tc}} \mathrm{O}_{2} \\
\text { (torr) }\end{array}$ & $\begin{array}{l}66.3 \text { (SD 11.5) } \\
(50-113)\end{array}$ & $\begin{array}{l}72.9 \text { (SD 15.0) } \\
(46-130)\end{array}$ & $\begin{array}{l}62.7 \text { (SD 15.3) } \\
(40-104)\end{array}$ \\
\hline $\mathrm{HR}$ & $\begin{array}{l}122.3 \text { (SD 1,3.4) } \\
(92-161)\end{array}$ & $\begin{array}{l}135.0(\mathrm{SD} 21.6) \\
(107-168)\end{array}$ & $\begin{array}{l}141.0(\text { SD 14.8) } \\
(77-177)\end{array}$ \\
\hline
\end{tabular}

* GA, gestation; BW, birthweight.

$\dagger$ A twin, GA 37 wk. gradually increasing increments according to the routine of the ward.

During the monitoring session, a multivariable recording sample lasting approximately $5 \mathrm{~min}$ was collected when the infant was in quiet sleep on the basis of the clinical criteria of Prechtl (11). Each baby was studied during the 1st, 3rd, and 5th day of life. The range of the age at the recording was 1 to $115 \mathrm{~h}$ of postnatal life.

$P_{\mathrm{tc}} \mathrm{O}_{2}$ and $P_{\mathrm{tc}} \mathrm{CO}_{2}$ monitoring. Continuous measurement of $\mathrm{P}_{\mathrm{tc}} \mathrm{O}_{2}$ and $\mathrm{P}_{\mathrm{tc}} \mathrm{CO}_{2}$ was performed $\left(\mathrm{TCO}_{2} \mathrm{M} 818\right.$, Novametrix Medical System, Wallingford, CT) with the skin electrodes placed on the upper thorax of the infant. The $\mathrm{O}_{2}$ electrode was on the right i.e. preductal and the $\mathrm{CO}_{2}$ electrode on the left. The $\mathrm{O}_{2}$ electrode was calibrated at zero and atmospheric $\mathrm{PO}_{2}$ immediately before attachment to the skin. The $\mathrm{CO}_{2}$ electrode was calibrated using 5 and $10 \% \mathrm{CO}_{2}$ mixtures at atmospheric pressure according to the recommendation of the manufacturer. $\mathrm{P}_{\mathrm{tc}} \mathrm{CO}_{2}$ and $\mathrm{P}_{\mathrm{tc}} \mathrm{O}_{2}$ electrodes were heated and maintained at $41^{\circ} \mathrm{C}$ and $43.5^{\circ} \mathrm{C}$, respectively, during the recordings. The mounted probes were allowed to stabilize for $20 \mathrm{~min}$ before the readings were considered valid. Visual display was used to verify the steady state. Steady state levels of the transcutaneous gas values were correlated with HRV and respiratory activity. The values were expressed as torr units ( $\mathrm{mm} \mathrm{Hg}$ ).

$H R$ and respiratory waveform monitoring. The electrocardiograms and TEZ waveforms were recorded by Corometrics 512 Neonatal Monitor (Corometrics Medical Systems, Wallingford, CT) from a bipolar lead, using recording electrodes at the V5 position bilaterally and earth lead in the right thigh. The recording was made when the transcutaneous $\mathrm{PO}_{2}$ and $\mathrm{PCO}_{2}$ values were stationary. The quality of the signals was visually verified, and the signals were simultaneously recorded on a magnetic tape (Racal Thermionic Store 4ds, Racal Recorders Ltd, Southampton, UK).

Computer processing of IHR and TEZ signals. The tape records were replayed, and a noise-free sample of 200 successive $R R$ intervals was obtained. The RR intervals were recognized
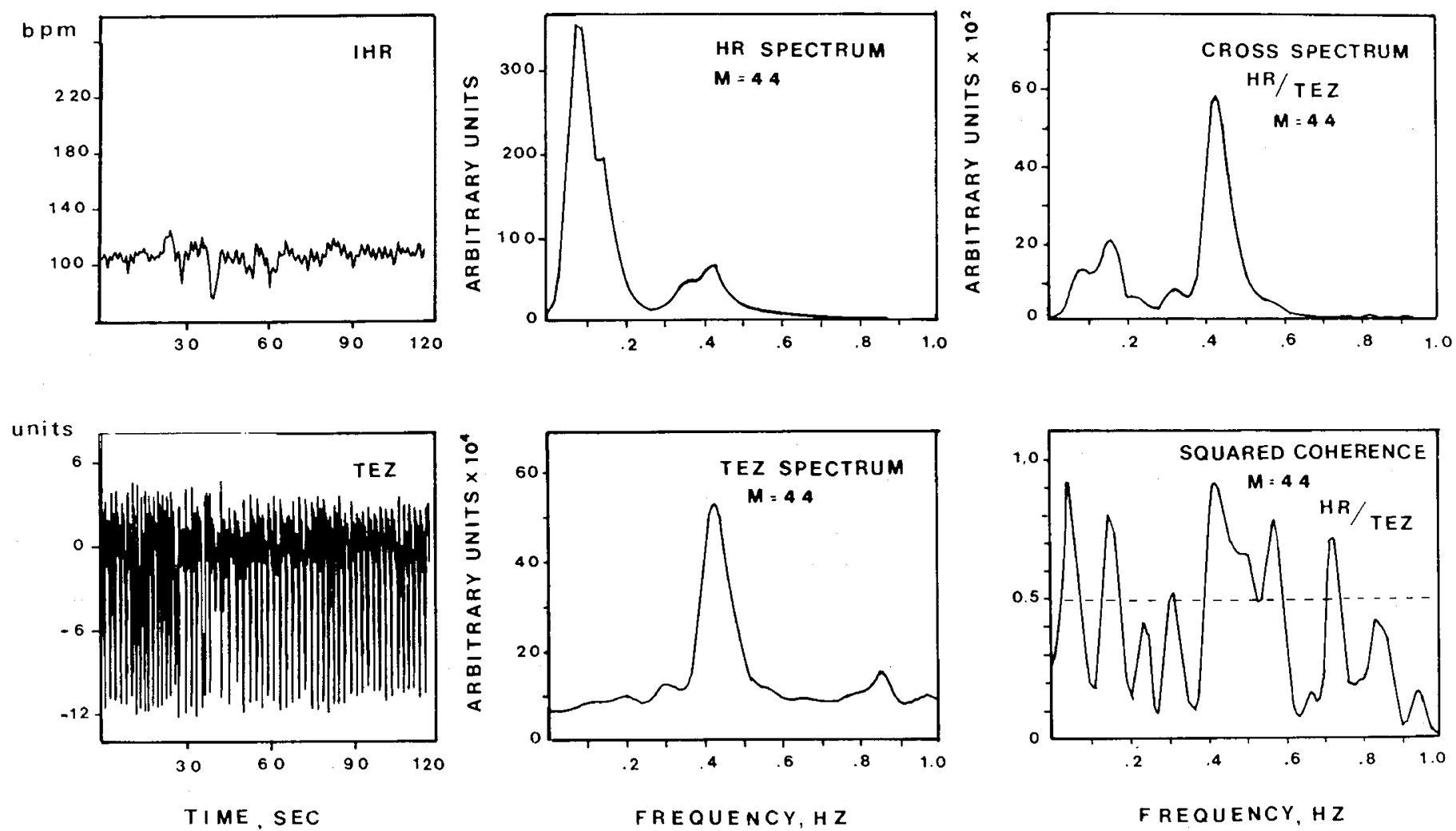

Fig. 1. IHR and TEZ signals and their FFT spectra. In this term baby, a peak of RSA is visible in HRV spectrum as well as in the cross-spectrum of the two variables. 
with an analogue $\mathrm{R}$ wave detector based on band-pass filtering and voltage treshold trigger (12). The pulses were then fed through an interval measurement interface, the RR intervals were measured (accuracy $2 \mathrm{~ms}$ ) and stored in digital form on a disc using a laboratory computer (Nova 3, Data General Corporation, Southboro, MA). Further analysis was carried out in a faster minicomputer (Eclipse C/150, Data General Corporation, Southboro, MA). The measured RR intervals from the electrocardiogram were converted to instantaneous HR. For spectral analysis, the IHR data were made continuous by linear interpolation and then sampled at $2.2 \mathrm{~Hz}$ and filtered using a linear digital filter $(\mathrm{Fc}, 0.025 \mathrm{~Hz})$.

The respiratory TEZ signal was digitized at a sampling rate of $16.6 \mathrm{~Hz}$ after low-pass filtering (by mode of MAXFLAT by JF Kaiser, Ref. 13) with $2 \mathrm{~Hz} \mathrm{Fc}$ to avoid aliasing, i.e. a high frequency "impersonating" a low frequency, before analogue/ digital conversion (Nova 3 and Eclipse C/150 computers, Data General Corp., Southboro, MA). The TEZ variability was first examined by plotting the digitized signal around a 0 mean (Fig. 1). Prior to spectral analysis, the signal was high-pass-filtered using a Hanning window of 99 data points with $0.02 \mathrm{~Hz} \mathrm{Fc}$ (by mode of WINDOW by LR Rabiner and CA McConegal, Ref. 13). Autocovariance function was then computed for each IHR signal and respirogram, and this was used to compute the powerspectrum using FFT to cover the frequency range of 0.02 to $1.0 \mathrm{~Hz}(14)$.

Event-by-event interactions between IHR and respiration were assessed by cross-spectral analysis. For the cross-spectral study, cross-covariance function was computed between the IHR and TEZ signals to identify common frequencies. In the computation of powerspectra and cross-spectra for the IHR and TEZ signals autovariance-covariance method by means of Eclipse $C / 150$ computer was applied (14). The value of truncation points (M), which displays the number of summations needed for one point in the spectrum, was empirically selected to be 44 . Coherence spectra were computed between the source signals to assess the significance of peaks in the cross-spectral density. Cross-spectral peaks with spectral coherence $>0.5$ were considered significant.

The individual powerspectra were finally band-integrated (15). The band width was $0.05 \mathrm{~Hz}$. Average spectral densities for each group were used for intergroup comparisons of spectral patterns. The Wilcoxon nonparametric test was used in this comparison. IHR spectral and cross-spectral data of IHR and TEZ were then examined in relation to respective values of $\mathrm{P}_{\mathrm{tc}} \mathrm{O}_{2}$ and $\mathrm{P}_{\mathrm{tc}} \mathrm{CO}_{2}$ by means of linear correlation analysis.

\section{RESULTS}

In all the neonates, most of the HRV power was at the LF $<$ $0.2 \mathrm{~Hz}(<12 \mathrm{oscillations} / \mathrm{min})$. The LF spectral density of HRV $(<0.2 \mathrm{~Hz})$ was higher in the term babies compared to the healthy preterm babies on day 3 , but there were no differences on the other days studied. In these babies, the LF spectral density increased postnatally. In the babies with RDS, both LF and high frequency $(>0.2, \mathrm{HF}) \mathrm{HRV}$ density were very low throughout the study (Fig. 2).

There were differences in the distribution of spectral components of HRV between the three groups (Fig. 3): In the term babies, the peak spectral density of HRV was at $<0.1 \mathrm{~Hz}$ on days 1 and 3 and at $0.15 \mathrm{~Hz}$ on day 5 . In the preterm babies, the peak activity was at $<0.1 \mathrm{~Hz}$ on all days; on day 3 the spectrum had two peaks. In these healthy babies, the major activity was in LF area, and the HRV spectral pattern finally became unimodal in appearance. The babies with RDS had an entirely different spectral distribution pattern. On day 1 , three peaks of HRV were seen; the spectral density was trimodal. The highest peak (or mode) value was at $0.15 \mathrm{~Hz}$ on day $1,0.10 \mathrm{~Hz}$ on day 3 , and $<$ 0.10 on day 5 . There were two additional peaks at 0.35 to 0.45
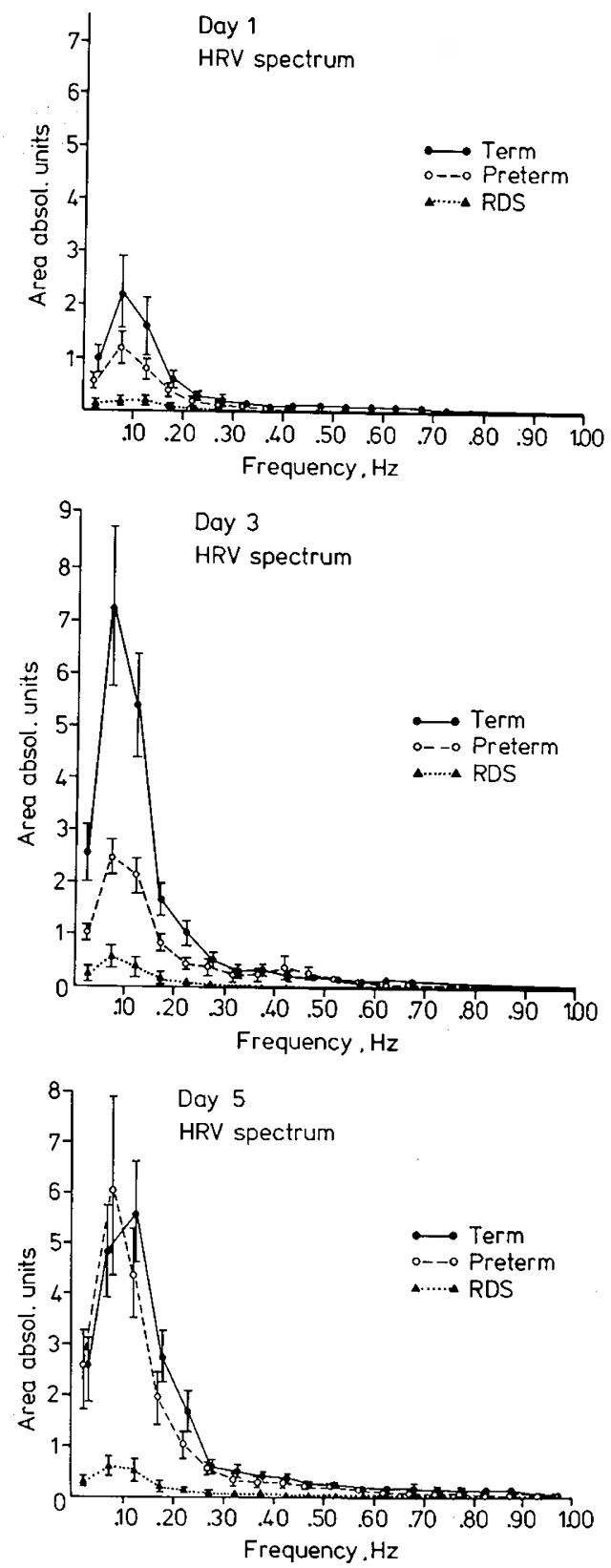

Fig. 2. The absolute spectral densities of HRV on days 1,3 , and 5 in healthy term, preterm, and RDS infants. The LF density of healthy term and preterm babies differs on day 3 . The spectral density of babies with RDS is small.

$\mathrm{Hz}$ and at 0.65 to $0.8 \mathrm{~Hz}$ on days 1 and 3 . With increasing postnatal age, the distribution finally became unimodal, with the peak at frequencies $<0.1 \mathrm{~Hz}$. In other words, the pattern was transformed and finally resembled that of the healthy preterm babies, but the total HRV (area under the spectral curve) remained abnormally low.

The respiratory amplitude spectrum was computed in percentage scale because absolute variabilities of TEZ signals are difficult for intergroup comparison. The spectrum was fairly flat, i.e. evenly distributed periodicities were present over the frequency area $<1 \mathrm{~Hz}$ in the term babies. On day 5, a peak appeared at $0.55 \mathrm{~Hz}$; it corresponded to the frequency of visually counted average RR. In the healthy preterm babies, there was a peak in the LF region on all days. This peak was most prominent on day 1 . In the babies with RDS, the peak of ventilator frequency was clearly identifiable in the fairly flat spectrum of spontaneous periodic components. Finally, the respiratory vari- 

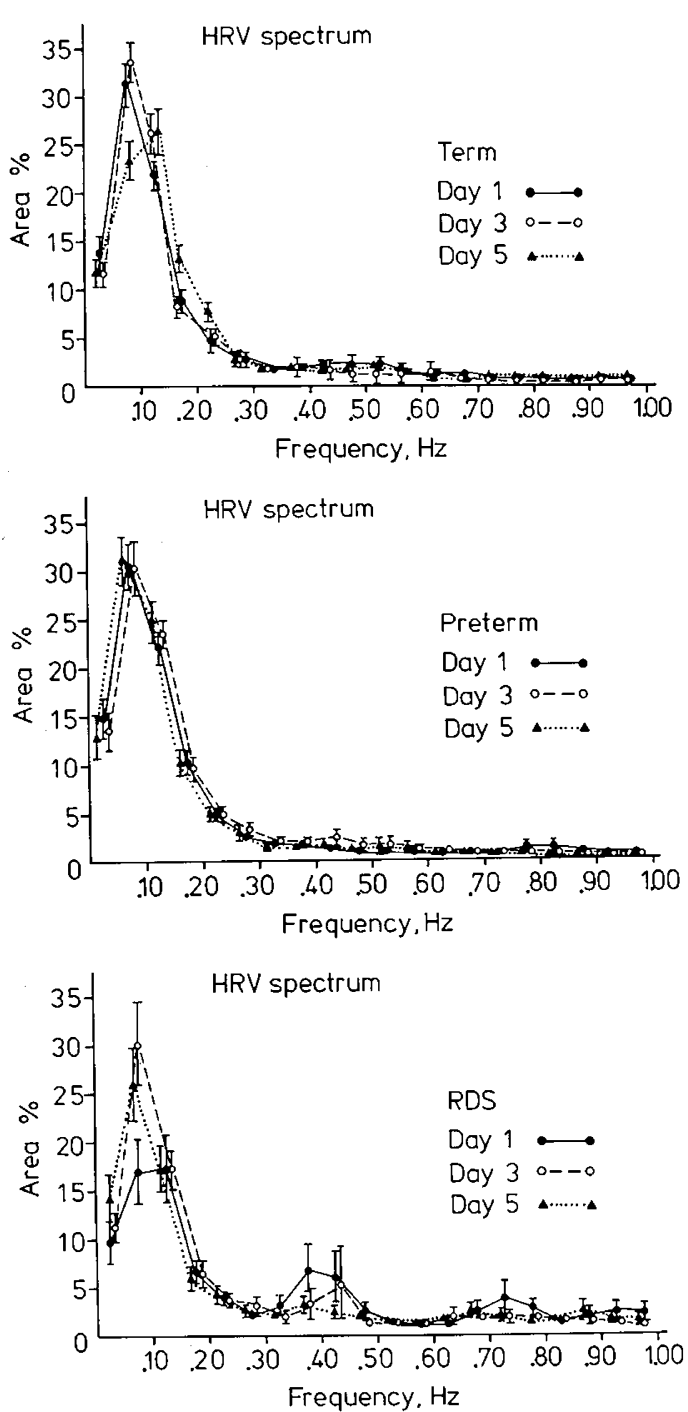

Fig. 3. The distribution of spectral densities of HRV presented in percentage units. In healthy term babies, the LF density peak moves forward. The spectral density of healthy preterm babies remains static. In RDS, the density accumulates to LF region on day 5 .

ability accumulated in the LF area in the babies with RDS (Fig. 4).

The distribution of the cross-spectral density changed from a unimodal LF pattern to a bimodal pattern in the term babies. In the healthy preterm infants, the unimodal LF pattern persisted. In the babies with RDS, the cross-spectral density was trimodal, and with increasing postnatal age the relative power in LF area increased and that in HF area decreased (Fig. 5).

Significant cross-spectral activity peaks between HRV and TEZ spectrum were most prominent in healthy and sick preterm babies, of whom approximately $80 \%$ had significant common cardiorespiratory periodicities in these two signals on all days studied. In the term babies, this figure fell from approximately $80 \%$ on day 1 to approximately $50 \%$ on days 3 and 5 . The presence of LF and HF cross-spectral activity was investigated in babies who displayed significant coherence in cross-spectral analysis. LF cross-spectral activity occurred in 55 and $38 \%$ of the term babies on days 1 and 3, respectively, and then diminished to $5 \%$ on day 5 , when the cross-spectral activity preponderance was in the HF region. Of the preterm babies, $40 \%$ had significant cross-spectral LF activity that did not diminish during the study. In healthy preterm babies, it rose to a peak of approximately $60 \%$ on day 3 .
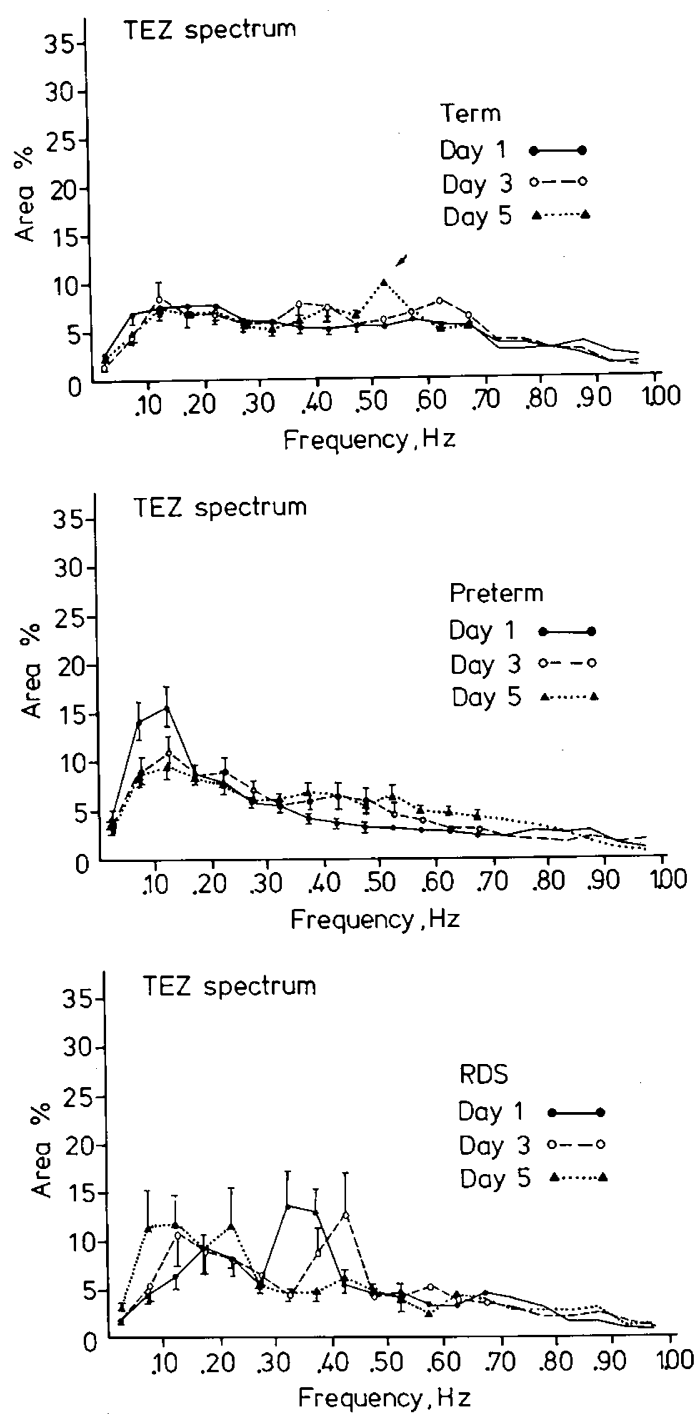

Fig. 4. The respiratory amplitude spectrum on days 1,3 , and 5 in the three groups. Distribution of power in percentage scale. The term babies have a peak on day 5 , which indicates an emergence of a major periodicity in respiration $0.53 \mathrm{~Hz}=32 \mathrm{~min}^{-1}$. In babies with RDS a ventilator induced peak is visible on days 1 and 3 .

There was no significant correlation between the transcutaneous values of $\mathrm{PO}_{2}$ and $\mathrm{PCO}_{2}$ and the components of the IHR spectral and the cross-spectral density.

\section{DISCUSSION}

Spectral approach allows more detailed analysis of the slow HRV with the exploration of new hypothetical control mechanisms. Because the area of the spectrum represents the squared $\mathrm{SD}$ of $\mathrm{HR}$, it gives information about the magnitude of the various frequencies of overall variability. The power spectrum of HRV in healthy term neonates was found to contain mainly LF components, as in previous studies $(9,10,16,17)$. Baroreceptor control is displayed in the LF area (18). Interestingly, Giddens and Kitney also propose chemoreceptor activity in very LF area on the basis of circulatory times (9). The reninangiotensin system (1) and vasomotor thermoregulation (15) have been demonstrated to act on HR in the LF area. Dykes et al. (16) have studied HRV and respiration in healthy term babies by spectral analysis and defined three different areas $(<0.02,>0.02-0.2$, and $>0.2$ ) in both signals, The very LF area $<0.02 \mathrm{~Hz}$ was cut out in our study due to the filtering methods. The HRV spectral 

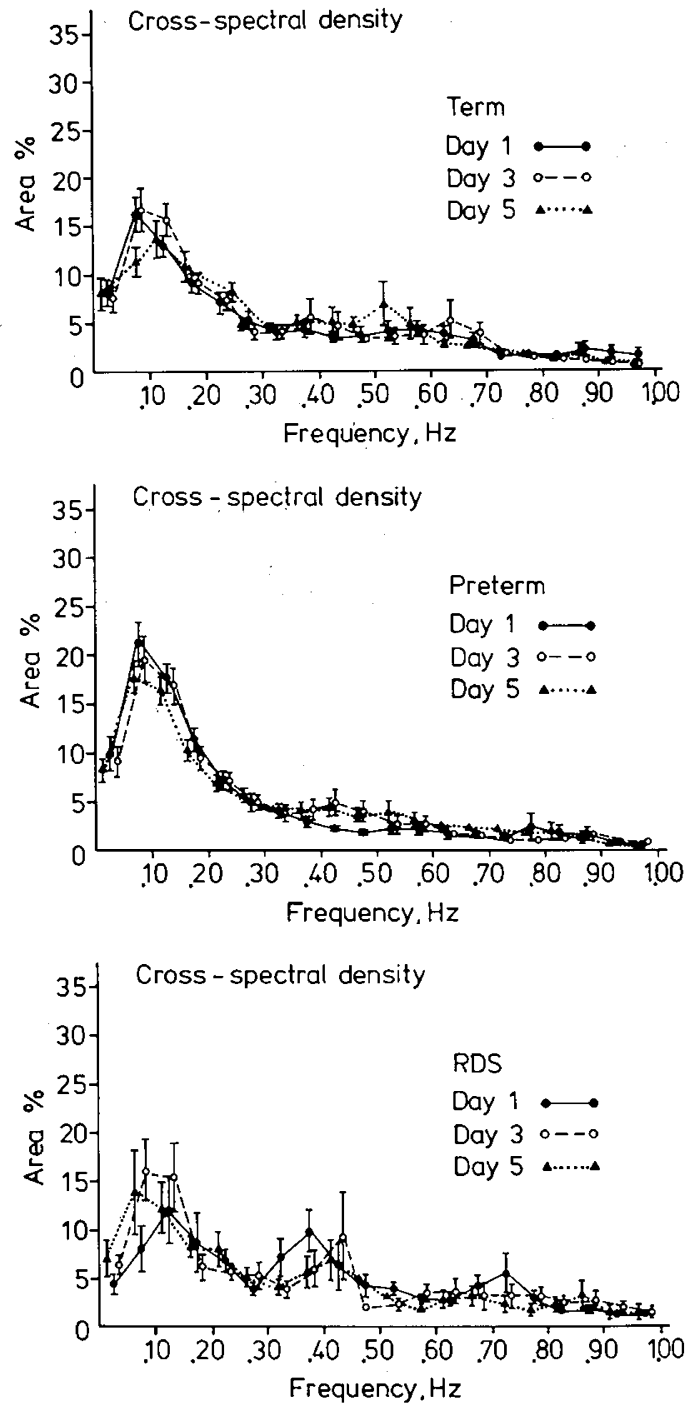

Fig. 5. The cross-spectral activity between HRV and TEZ spectra. In term babies the cross-spectral activity decreases in LF area and increases in HF area where RSA peak becomes visible on days 3 and 5. In healthy preterm babies, RSA activity is not identifiable. In babies with RDS, ventilator-induced RSA peaks are seen on days 1 and 3 .

density seemed to have a static pattern in healthy preterm babies. The pattern was similar to the pattern of the healthy term babies on day 1 . However, in the term babies on later days, there was a shift of LF peak to the right, in other words, the LF oscillation became faster. This might be related to increased sensitivity of regulatory receptors or to increased conductivity in the afferentefferent feedback loop of the system. It might also be a sign of an increase in the activity of the renin-angiotensin system in term babies. The renin-angiotensin system has a suppressive effect on the slow oscillations of LF activity (19). In term babies, there was also an increase of activity in the HF area postnatally, i.e. the appearance of oscillations $>0.2 \mathrm{~Hz}\left(>12 \mathrm{~min}^{-1}\right)$ (Fig. 1). Thus there was a time-related dynamic change in the appearance of $\mathrm{HF}$ activity which was related to maturity. This change might indicate appearance of RSA in this region when regular breathing becomes organized.

There was no correlation between the magnitude of the LF activity and transcutaneous values of $\mathrm{PO}_{2}$ or $\mathrm{PCO}_{2}$. The variability induced by chemoreceptors should visualize in this frequency area. The lack of correlation might be explained by the steady state situation in the recording, presuming that the activation of chemoreceptors is sensitive to changes rather than to levels of blood gases, or perhaps, the method used was too insensitive to detect the activation of chemoreceptors from the background activity.

In the babies with RDS, the trimodal pattern of HRV is interesting. The LF peak was the largest and thus comparable to the pattern of healthy babies. The second peak (HF area) obviously represents the RSA caused by extremely regular artificial ventilation. The third peak almost certainly represents the first harmonic of the ventilator frequency and is related to the square shape of the intermittent mandatory ventilation pulse.

Regular respiration is one of the criteria of quiet sleep (11). In these newborn babies, spectral analysis of respiratory electric impedance displayed a respiratory pattern with a considerable scatter of different periodic components of respiratory activity. In the healthy term babies, on days 3 and 5 , a pattern was seen where there was a peak at the area of visually verified $R R$. This means the emergence of a more regular breathing pattern. The peak moved to a lower frequency area with increasing postnatal age, i.e. the regular breathing slowed down. Thus the pattern of respiration in quiet sleep seems to be related to the maturity and postnatal age (age of continuous respiration) in newborn babies. There was no significant correlation between the RR and transcutaneous values of $\mathrm{O}_{2}$ or $\mathrm{CO}_{2}$ in this study.

In "classic" periodic breathing, one would expect two clusters of peaks, one at low frequency (frequency of periodicity) and the other at a higher frequency (frequency of average RR) area. The more even the distribution in frequencies, the more respiratory activity contains different periodicities. If $R R$ is regular, the spectrum displays only one peak and thus there is no other periodicity. This type of respiration has been used in investigation of RSA when, in artificial laboratory circumstances, voluntarily fixed $\mathrm{RR}$ induces one single peak in the spectrum. According to Giddens and Kitney (9), RSA in adults increases when the RR approaches $0.1 \mathrm{~Hz}\left(6 \mathrm{~min}^{-1}\right)$, i.e. the natural frequency of baroreceptor control loop, because of entrainment. Neonatal RR is of course far beyond $0.1 \mathrm{~Hz}(0.2-2 \mathrm{~Hz}$ in our study). In any case, under normal physiologic circumstances, RSA must be displayed in a wide area of different frequencies of heart rate, and thus its identification must involve an analysis of both HRV and respiratory spectra together.

In this study, the LF cross-spectral density was highest in preterm babies. Thus it appears that a major part of their HRV was related to respiration. Vallbona et al. (20) postulated that each breath results in arrival of numerous volleys of impulses to the cardioregulatory centers. If the frequency of feedback approaches the inherent free oscillation of the center, entrainment or synchronization of the rhythms occurs. This type of entrainment might function more effectively in a less mature and thus more simple system and explain our finding. On day 5, LF crossspectral density decreased and the incidence of LF cross-spectral peaks was reduced to one-tenth in term babies who, perhaps by then, had recruited a variety of regulative systems for adequate cardiorespiratory function. The preterm babies remained in a stationary phase where cardiorespiratory control in the LF area was interlocked. This situation might reduce their capacity of adjustment in this stage of incomplete adaptation.

In the term babies, the significant cross-spectral activity was mainly in HF area on day 5. As RSA caused by regular respiration visualizes in this area, this would mean the evolution of RSA as one component of HRV postnatally in term babies. In unventilated preterm babies, HF activity did not change in the same way.

One of the factors in the decreased HRV in babies with RDS might relate to the decreased compliance of the sick lung. The stiff lung creates an environment where respiratory excursions are small and thus the mechanical respiratory effect on the heart as a pump is small. This would minimize the possible effect of the respiratory push-pull movement on hemodynamics as one cause of HRV. Also the high spontaneous RR in RDS is likely to make the interaction between $\mathrm{HR}$ and respiration subtle, as 
Giddens and Kitney (9) propose. Accordingly, the time-related increase in the HF area of HRV spectrum of healthy infants was not seen in RDS in this study.

We conclude that during the first 5 days of postnatal life the term babies display a change in HRV and respiratory spectrum. In term babies, the LF cross-spectral density between HR and respiration decrease with advancing days, and a peak of RSA emerges in the HF area. The postnatally increasing LF HRV is then due to factors not connected with respiration, a change which probably reflects an increased ability to meet acute env1ronmental stress situations and is due to recruitment of a variety of regulatory mechanisms during postnatal adaptation. In healthy preterm infants, the HRV spectrum remains stationary. The development of regularity of respiration as displayed in the spectrum is also delayed in healthy preterm babies. In RDS there is a shift in the spectral distribution of HRV to the LF area. In preterm babies, the LF cross-spectral activity of HR and respiration does not change during the first 5 days of life, and in preterm babies with spontaneous respiration the $\mathrm{HF}$ area is flat. Thus LF HRV is very much connected with respiration in preterm babies who, by contrast, do not have a regularity of respiration to display conventional RSA in HF area. This holds especially in babies with RDS, in whom the absolute HRV is small. The tight interconnection of HR and respiration in LF area of preterm babies means restriction of mechanisms for adaptation compared with the term babies.

\section{REFERENCES}

1. Akselrod S, Gordon D, Ubel FA, Shannon DC, Barger AC, Cohen RJ 1981 Powerspectrum analysis of heart rate fluctuation. A quantitative probe of beat-to-beat cardiovascular control. Science 213:220-222

2. Pomeranz B, Macaulay RJB, Caudill MA, Kutz I, Adam D, Gordon D, Kilborn KM, Barger AC, Shannon DC, Cohen RJ, Benson H 1985 Assessment of autonomic function in humans by heart rate spectral analysis. Am J Physiol 248:H151-153

3. Valimaki I, Antila H, Antila K, Kero P 1980 Spectral analysis of neonatal heart rate. A preliminary report. In: Rolfe P (ed): Fetal and Neonatal Physiological Measurements, Pitman Press, Bath, England, pp 104-109

4. deBoer RW, Karemaker JM, Strackee J 1985 Relationships between shortterm blood-pressure fluctuations and heart-rate variability in resting subjects I: a spectral analysis approach. Med Biol Eng Comput 23:352-358

5. Rudolph AJ, Vallbona C, Desmond MM 1965 Cardiodynamic studies of the newborn III. Heart rate patterns in infants with idiopathic respiratory distress syndrome. Pediatrics 36:551-559

6. Tarlo PA, Valimaki I Rautaharju PM 1971 Quantitative computer analysis of cardiac and respiratory activity in newborn infants. J Appl Physiol 31:7075

7. Jenkins JG 1980 The study of heart rate variability in the newborn. $\mathrm{PhD}$ thesis, Queen's University, Belfast

8. Kero P 1974 Heart-rate variation in infants with the respiratory distress syndrome. Acta Paediatr Scand [Suppl]250:1-70

9. Giddens DP, Kitney RI 1985 Neonatal heart rate variability and its relation to respiration. J Theor Biol 113:759-780

10. Anttila H, Valimaki I, Gadzinovski J, Oja R, Antila K, Kero P 1986 Spectral components of heart rate variability in normal neonates and in infants with respiratory distress. In: Copeland $\mathrm{K}$ (ed): Progress Reports on Electronics in Medicine and Biology, IERE, London, pp 319-324

11. Prechtl HFR 1974 The behavioral states of the newborn infant. (A review). Brain Res 76:185-212

12. Antila K 1979 Quantitative characterization of heart rate during exercise. Scand J Lab Inv Suppl 153

13. Programs for digital signal processing 1979 Digital Signal Processing Committee, IEEE Acoustic, Speech and Signal Processing Society (eds). IEEE Press, New York

14. Jenkins GM, Watts DG 1969 Spectral analysis and its applications.

15. Lindqvist A, Oja R, Hellman O, Valimaki I 1983 Impact of thermal vasomotor control on the heart rate variability of newborn infants. Early Hum Dev 8:37-47

16. Dykes FD, Ahmann PA, Baldzer K, Carrigan TA, Kitney R, Giddens DP 1986 Breath amplitude modulation of heart rate variability in normal full term neonates. Pediatr Res 20:301-308

17. Kitney RI, Ong HG 1986 An analysis of cardiorespiratory control in babies and its relation to sudden infant death syndrome. Automedica 7:105-126

18. Kitney RI 1984 New findings in the analysis of heart rate variability in infants. Automedica 5:289-310

19. Gordon D, Cohen RJ, Kelly D, Akselrod S, Shannon DC 1984 Sudden infant death syndrome: Abnormalities in short term fluctuations in heart rate and respiratory activity. Pediatr Res 18:921-926

20. Vallbona C, Desmond MM, Rudolph AJ, Fisher Pap L, Hill RM, Franklin RR, Rush JB 1963 Cardiodynamic studies in the newborn II. Regulation of the heart rate. Biol Neonate 5:159-199 